\title{
Erratum to: The comparison of NN, SVR, LSSVR and ANFIS at modeling meteorological and remotely sensed drought indices over the eastern district of Isfahan, Iran
}

\author{
Iman Khosravi $^{1} \cdot$ Yaser Jouybari-Moghaddam $^{1} \cdot$ Mohammad Reza Sarajian $^{1}$
}

\section{Erratum to: Nat Hazards \\ DOI 10.1007/s11069-017-2827-1}

In the initial online-first publication the name of author Yaser Jouybari-Moghaddam was given as Yaser Jouybari Moghaddam (without the hyphen) which may have given rise to confusion about what the last name is. This has now been corrected.

The online version of the original article can be found under doi:10.1007/s11069-017-2827-1.

Iman Khosravi

iman.khosravi@ut.ac.ir

Yaser Jouybari-Moghaddam

y.joibari@ut.ac.ir

Mohammad Reza Sarajian

sarajian@ut.ac.ir

1 Department of Remote Sensing, School of Surveying and Geospatial Engineering, College of Engineering, University of Tehran, Tehran, Islamic Republic of Iran 